\title{
Relationship between Angiogenic and Inflammatory Biomarkers and Diabetic Retinopathy
}

\author{
C. Domngang Noche ${ }^{*}$, F. Nzoundji Kouosseu', Y. Bilong2, P. Chuisseu Djamen', \\ P. Fotsing Kwetche ${ }^{1}$, A. Noutchie Lowe ${ }^{1}$, B. Agoons ${ }^{2}$, M. Temgoua ${ }^{2}$, J. C. Katte Djabou ${ }^{2}$, \\ E. Sobgwi ${ }^{2}$
}

${ }^{1}$ Higher Institute of Health Sciences, Université des Montagnes, Bangangté, Cameroon

${ }^{2}$ Faculty of Medicine and Biomedical Sciences, Université de Yaounde 1, Yaounde, Cameroon

Email: ${ }^{*}$ dockrystlnoche@gmail.com

How to cite this paper: Noche, C.D., Kouosseu, F.N., Bilong, Y., Djamen, P.C., Kwetche, P.F., Lowe, A.N., Agoons, B., Temgoua, M., Katte Djabou, J.C. and Sobgwi, E. (2020) Relationship between Angiogenic and Inflammatory Biomarkers and Diabetic Retinopathy. Open Journal of Ophthalmology, 10, 44-54.

https://doi.org/10.4236/ojoph.2020.101006

Received: November 25, 2019

Accepted: January 3, 2020

Published: January 6, 2020

Copyright $\odot 2020$ by author(s) and Scientific Research Publishing Inc. This work is licensed under the Creative Commons Attribution International License (CC BY 4.0).

http://creativecommons.org/licenses/by/4.0/

\begin{abstract}
Background: Diabetic retinopathy (DR) is a common complication of diabetes mellitus and a major cause of vision loss in the working age population. Its pathogenesis is poorly understood but may involve low grade chronic inflammation and angiogenesis. The aim of this study was to evaluate the relationship between serum levels of one inflammatory (IL-6) and angiogenic cytokine (VEGF-A) with the presence and severity of DR in type 2 diabetic mellitus patients. Methods: From January to June 2019, we conducted a cross-sectional analytical study on 84 patients out of which 31 developed DR and 53 did not. All patients underwent complete ophthalmological examination and laboratory analysis for IL-6 and VEGF-A with ELISA Technique. We studied the relation of IL- 6 and VEGF-A with the presence and severity of DR, HBA1c, the duration of diabetes. Results: The group with DR had statistically significant higher levels of VEGF-A compared to the control group (390.5 $\mathrm{pg} / \mathrm{ml}$ vs. $173.1 \mathrm{pg} / \mathrm{ml} ; \mathrm{p}=0.007)$. There was no significant difference in IL-6 levels between both groups ( $42.8 \mathrm{pg} / \mathrm{ml}$ vs. $31.7 \mathrm{pg} / \mathrm{ml} ; \mathrm{p}=0.10)$. Equally there was no association between these 2 cytokines and macular oedema or with the severity of DR. The level of IL- 6 was associated to the balance of diabetes ( $p=$ $0.006)$ although VEGF-A was not $(\mathrm{p}=0.15)$. Moreover, Il-6 $(\mathrm{p}=0.31)$ and VEGF-A ( $p=0.24)$ were not linked to the duration of diabetes. Conclusion: Serum concentrations of VEGF-A have an effect on the development of DR. They correlate with the presence of the disease but IL- 6 does not. However, IL- 6 was associated to the balance of diabetes. These 2 biomarkers may play a role in the pathophysiology of diabetes and the diabetic retinopathy. Findings on Il-6 and VEGF-A may therefore contribute to the development of the diagnosis tools and caretaking of diabetes and diabetic retinopathy.
\end{abstract}




\section{Keywords}

Retinopathy, Type 2 Diabetes Mellitus, Vascular Endothelial Growth Factor-A, Interleukine-6, Cytokines

\section{Introduction}

Diabetic retinopathy (DR) is one of the most common microvascular complications in patients with type 2 diabetes mellitus. About $8.8 \%$ of the population suffers from diabetes mellitus out of which at least one-third develop DR at different stages [1] [2]. DR is the leading cause of vision loss in working-age adults and the fifth leading cause of reversible blindness across the world [3] [4]. Like in other African countries, the prevalence of DR seems to be high in Cameroon [5] [6] [7]. The available treatments for DR (laser photocoagulation, intravitreal injections of corticosteroids) are only indicated in the advanced stages of the disease [8]. These treatments are also associated with significant adverse effects [9]. The control of risk factors (blood glucose, high blood pressure) is the only therapeutic tool that practitioners can use in the early stages of the disease. However, these risk factors did not account for all the risk for development and progression to sight-threatening diabetic retinopathy [10]. It becomes therefore evident that other elements of the diabetic milieu are involved in the pathology. This is why research focuses on the pathophysiological mechanisms of DR for the development of new strategies. Although this pathophysiology is not yet elucidated, several phenomena likely play an important role in the pathology, including inflammation and angiogenesis. Studies based on intraocular fluids confirmed the hypothesis that pro-inflammatory and pro-angiogenic cytokines are associated with the progression of DR [11] [12]. Serum investigation represents here one of the least expensive and invasive alternatives. Moreover, with the expected increase in the number of people living with diabetes in 2045 [1], there is an urgent need to find new diagnostic and therapeutic tools for DR. In this regards, serum evaluation of angiogenic and inflammatory biomarkers may be useful in the early detection of the disease, in identifying patients at risk of developing proliferative diabetic retinopathy (PDR) and in monitoring the efficacy of new drugs. Circulating VEGF has been reported to be an important factor involved in the development of the diabetic retinopathy [13] [14] [15]. Moreover, there is an association between inflammatory proteins as TNF and interleukins [14] [15] and the development of microvascular complications of diabetes. However, there is a lack of studies on the relation between circulating cytokines and the progression of diabetic retinopathy in Sub-Saharan Africa. The aim of this present study was to analyze the relationship between the serum level of an angiogenic (VEGF-A) and a inflammatory (IL-6) biomarkers and the presence and severity of diabetic retinopathy (DR) in patients with type 2 diabetes mellitus. 


\section{Methods}

\section{Design and subjects}

This prospective analytical study was carried out from January to June 2019, at the National Obesity Centre of the Central Hospital of Yaoundé and at INNEL Medical Centre. It was approved by the Centre Regional Ethics Committee for Human Health Research ( ${ }^{\circ}$ 0058/19/AR/MINSANTE/SG/DHCY/CM/SM) and from the institutional review board of Université des Montagnes $\left(\mathrm{N}^{\circ}\right.$ 2019/104/UdM/PR/CIE). Written informed consent was obtained from each patient prior to enrolment.

The study population was composed of type 2 diabetes mellitus patients followed up at the Central Hospital of Yaoundé. We excluded pregnant women and all individuals having acute and chronic ophthalmological disorders such as glaucoma, cataract, age-related macular degeneration, occlusion of retinal artery or vein. The Whitley formula was used for the sample size calculation. Our primary outcome was the median difference in VEGF-A in the two groups.

A detailed history on diabetes, its chronic complications and other comorbidities were recorded for all participants. The body height and weight were recorded and the body mass index calculated. Blood pressure was measured in all participants in the sitting position after 10 minutes. All patients underwent a complete ophthalmological examination including visual acuity measurement and fundus examination. The best corrected visual acuity was measured using the Monoyer scale.

\section{Fundus findings}

After pupil dilation using tropicamide 5\% and phenylephrine 5\% eye drops, diagnosis of DR was performed by fundus examination with a slit lamp and a 78 $\mathrm{D}$ lens. Moreover, a colour fundus photography was carried out using a Topcon non mydriatic camera. Staging of DR was performed according to the International Clinical Diabetic Retinopathy Disease Severity Scale of the American Academy of Ophthalmology (AAO) [16]. The presence of a macular oedema was also noted.

Patients with diabetic retinopathy were thereafter classified in two groups (non-proliferative DR and proliferative DR). They were staged according to the presence and the severity of DR of the more affected eye.

\section{Blood Collection}

Blood samples were collected before midday in dry tubes. Sera were separated by centrifugation within 45 minutes after collection. For each participant, the serum $(0.5 \mathrm{ml})$ was collected in two Eppendorf tubes and kept frozen at $-20^{\circ} \mathrm{C}$ before analysis.

\section{Determination of VEGF-A and IL-6}

Analysis of VEGF-A and IL- 6 was carried out by immunoenzymatic ELISA method according to the manufacturer protocol (Elabscience, USA). We used the following kits:

- Human VEGF-A ELISA kit (lot No SU5YE6RU5L, Elabscience, USA) and 
- Human IL-6 ELISA Kit (lot No 8SJ2I6UYFE, Elabscience, USA).

\section{Statistics}

Data was analysed using EPI Info statistical program version 7.2.2.1. Results are expressed as median [range] for continuous variables, and as frequencies for categorical variables. Values with abnormal distribution were analysed using the Mann-Whitney and Kruskal Wallis test. T test and ANOVA were used to analyze values with normal distribution. Multiple logistic regression analysis was conducted to assess independent risk factors for diabetic retinopathy; associations were calculated as ORs (odds ratios) with the corresponding 95\% confidence intervals (CIs). A "p value" of less than 0.05 was considered as statistically significant.

\section{Results}

\section{General characteristics}

Eighty-four patients ( 31 in the DR group and 53 in the control group) were enrolled in this study ( 39 male and 45 female). Table 1 summarizes the clinical and biological characteristics of the type 2 diabetes patients according to the presence or the absence of diabetic retinopathy.

The median age in the DR group was 60 (range 52 - 66) while in the control group it was 54 (range $47-60$ ). There were no significant differences with respect to demographic features including gender between the two groups. The median diabetes duration was 10 years (range 2 - 13) in the DR group and 4 (range 1 - 7) in the control group.

Table 1. Clinical and biological characteristics of participants.

\begin{tabular}{cccc}
\hline & DR $(\mathbf{n}=31)$ & Absence of DR $(\mathbf{n}=53)$ & P value \\
\hline Age (years) & $60[52-66]$ & $54[47-60]$ & 0.006 \\
Diabetes duration (years) & $10[2-13]$ & $4[1-7]$ & 0.01 \\
BMI (kg/m²) & $28.8[25-31.5]$ & $28.6[25.2-32.7]$ & 0.85 \\
SBP (mmHg) & $142[125-163]$ & $134[119-142]$ & 0.07 \\
DBP (mmHg) & $87[82-100]$ & $83[75-92]$ & 0.07 \\
HbA1C (\%) & $8.5[7.8-10.3]$ & $6.9[5.6-10.1]$ & 0.009 \\
BUN (g/l) & $0.3[0.3-0.4]$ & $0.3[0.2-0.3]$ & 0.04 \\
Creatinine (mg/l) & $11[8.6-16.5]$ & $9.4[8-11]$ & 0.01 \\
GFR (ml/mn) & $78.3[51-106.4]$ & $102.4[88.1-128]$ & 0.01 \\
Total cholesterol (g/l) & $1.8[1.7-2.1]$ & $1.6[1.5-1.9]$ & 0.04 \\
LDL cholesterol (g/l) & $1.1[0.9-1.4]$ & $1[0.8-1.1]$ & 0.09 \\
HDL Cholesterol (g/l) & $0.5[0.4-0.6]$ & $0.4[0.4-0.5]$ & 0.36 \\
Triacylglycerols (g/l) & $1.3[1-1.7]$ & $1.3[0.8-1.6]$ & 0.58 \\
\hline
\end{tabular}

Values are presented as median [range] DR: Diabetic retinopathy, BMI: Body mass index, SBP: Systolic blood pressure, DBP: Diastolic blood pressure, HbAlc: glycated haemoglobin, BUN: Blood urea nitrogen, GFR: glomerular filtration rate, LDL: low density lipoproteins, HDL: High density lipoproteins. 
The difference was significant for the following variables: 1) the duration of diabetes (DR Group: 10 years vs Control group: 4 years; $p=0.01$ ); 2) glycated haemoglobin (DR Group: 8.5\% vs. Control group: 6.9\%; $\mathrm{p}=0.009$ ); 3 ) the glomerular filtration rate (DR Group: $78.3 \mathrm{ml} / \mathrm{mn}$ vs. Control group: 102.4 $\mathrm{ml} / \mathrm{mn} ; \mathrm{p}=0.01$ ).

\section{Associated factors}

The research for associated factors was carried out (Table 2). The parameters related to diabetes (duration, imbalance), comorbidities (high blood pressure) and its complications (nephropathy, low visual acuity) were associated with diabetic retinopathy.

After a multiple logistic regression, only high blood pressure $(\mathrm{p}=0.01)$ and imbalance of diabetes $(p=0.005)$ were eventually associated to diabetic retinopathy as a risk factor (Table 3 ).

Table 2. Clinical and biological factors associated to diabetic retinopathy.

\begin{tabular}{ccccc}
\hline & $\begin{array}{c}\text { DR } \\
\mathbf{n}=\mathbf{3 1} \\
\text { Overall } \mathbf{n}(\%)\end{array}$ & $\begin{array}{c}\text { Control group } \\
\mathbf{n}=\mathbf{5 3} \\
\text { Overall } \mathbf{n}(\%)\end{array}$ & $\begin{array}{c}\text { Odds ratio } \\
\text { (CI à 95\%) }\end{array}$ & P value \\
\hline Age $>50$ years & $27(87.1)$ & $33(62.3)$ & $4.1(1.2-13.4)$ & 0.01 \\
Diabetes duration $>5$ ans & $22(71)$ & $19(35.6)$ & $4.4(1.7-11.4)$ & 0.001 \\
High blood pressure & $26(83.9)$ & $26(49.1)$ & $5.4(1.8-16.1)$ & 0.001 \\
Diabetic nephropathy & $8(25.8)$ & $2(3.8)$ & $8.8(1.7-45.1)$ & 0.004 \\
Low visual acuity $(<7 / 10)$ & $14(45.2)$ & $11(20.7)$ & $3.1(1.2-8.3)$ & 0.01 \\
HbA1C $\geq 7 \%$ & $25(80.6)$ & $24(45.3)$ & $5(1.7-14.3)$ & 0.001 \\
GFR $<90$ ml/mn & $15(48.4)$ & $16(30.2)$ & $2.2(0.8-5.4)$ & 0.09 \\
Dyslipidemia & $19(61.2)$ & $30(56.6)$ & $1.3(0.5-3.4)$ & 0.55 \\
Total Cholesterol $>2 \mathrm{~g} / 1$ & $8(25.8)$ & $9(16.9)$ & $1.7(0.5-5)$ & 0.33 \\
LDL $>1 \mathrm{~g} / 1$ & $13(41.9)$ & $22(41.5)$ & $1(0.4-2.5)$ & 0.96 \\
Triacylglycerol $>1.5 \mathrm{~g} / 1$ & $9(29)$ & $16(30.2)$ & $1.3(0.4-3.6)$ & 0.66 \\
HDL $<0.4 \mathrm{~g} / \mathrm{l}$ & $9(29)$ & $20(37.7)$ & $1(0.3-2.5)$ & 0.84 \\
\hline
\end{tabular}

DR: Diabetic retinopathy, CI: Confidence interval, HbAlc: glycated haemoglobin, GFR: glomerular filtration rate, LDL: low density lipoproteins, HDL: High density lipoproteins.

Table 3. Multiple logistic regression analysis of factors associated with diabetes retinopathy.

\begin{tabular}{ccc}
\hline Variables & Odds ratio (CI à 95\%) & P value \\
\hline Age $>50$ years & $1.3(0.3-5.9)$ & 0.75 \\
Low visual acuity & $3.1(0.8-5.6)$ & 0.10 \\
Diabetes duration $>$ 5years & $3.1(0.9-10.2)$ & 0.06 \\
High blood pressure & $5.9(1.4-25,1)$ & 0.01 \\
Diabetic nephropathy & $2.2(0.3-13.3)$ & 0.40 \\
HbA1C $\geq 7 \%$ & $5.9(1.6-20.6)$ & 0.005 \\
\hline
\end{tabular}

CI: Confidence interval, HbA1c: glycated haemoglobin. 


\section{Profile of cytokines}

The median serum level of IL-6 was 33.5 (range 7.9 - 50.6) while that of VEGF-A was 189.3 (range 112.2 - 411.1) as presented in Table 4.

There was a highly significant increase in serum VEGF-A in the diabetic retinopathy group compared to the control group ( $p=0.007$ ) while increase of IL-6 in the DR group was not significant when it was compared to the control group $(\mathrm{p}=0.09)$.

Concerning the severity, there was no association between these cytokines and the severity of diabetic retinopathy (Il-6: $\mathrm{p}=0.47$; VEGF-A: $\mathrm{p}=0.20)$ and with the presence of macular oedema (Il-6: $p=0.20$; VEGF-A: $p=0.84$ ). Circulating IL- 6 was associated to the balance of diabetes $(p=0.006)$ although VEGF-A was not $(\mathrm{p}=0.15)$.

Circulating Il-6 $(\mathrm{p}=0.31)$ and VEGF-A $(\mathrm{p}=0.24)$ were not linked to the duration of diabetes.

\section{Discussion}

Diabetic retinopathy is the leading cause of blindness in active people in developing countries [2] [3] [4]. Although inflammatory and angiogenic markers are

Table 4. Relation between cytokines and diabetes' parameters.

\begin{tabular}{|c|c|c|}
\hline & $\mathrm{Il}-6(\mathrm{pg} / \mathrm{ml})$ & VEGF - A (pg/ml) \\
\hline Median (range) & $33.5(7.9-50.6)$ & $189.3(112.2-411.4)$ \\
\hline Mean \pm Standard deviation & $51 \pm 72(7.8-399.2)$ & $267.1 \pm 198.8(31.2-748.8)$ \\
\hline \multicolumn{3}{|l|}{ Forms of diabetes } \\
\hline Diabetic retinopathy $(\mathrm{n}=31)$ & $42.8(7.8-53.8)$ & $390.5(134-572.7)$ \\
\hline Controls $(n=53)$ & $31.7(7.9-43.2)$ & $173.1(107.3-269.2)$ \\
\hline$P$ value & 0.09 & 0.007 \\
\hline Non proliferative diabetic retinopathy $(\mathrm{n}=28)$ & $44(7.8-54.7)$ & $421.3(125-572.7)$ \\
\hline Proliferative diabetic retinopathy $(\mathrm{n}=3)$ & $24.1(7.9-40.3)$ & $134.1(133.9-188.2)$ \\
\hline$P$ value & 0.47 & 0.20 \\
\hline Maculopathy $(n=10)$ & $43.2(30.2-50.5)$ & $186.2(86.2-360.5)$ \\
\hline No maculopathy $(\mathrm{n}=74)$ & $32.4(7.8-51.6)$ & $195.2(118.2-419)$ \\
\hline$P$ value & 0.20 & 0.84 \\
\hline \multicolumn{3}{|l|}{ Imbalance of Diabetes } \\
\hline $\mathrm{HbAlc}>7 \%$ & $42.4(27.8-58.5)$ & $249.3(134.1-458.8)$ \\
\hline $\mathrm{HbA} 1 \mathrm{C}<7 \%$ & $8.9(7.9-40)$ & $173.1(107.3-304.9)$ \\
\hline$P$ value & 0.006 & 0.15 \\
\hline \multicolumn{3}{|l|}{ Duration of diabetes } \\
\hline Diabetes duration $\geq 10$ years & $35.7(7.8-53.8)$ & $249.3(134.1-458.8)$ \\
\hline Diabetes duration $<10$ years & $20.1(7.9-45.5)$ & $176.6(112.2-342.7)$ \\
\hline$P$ value & 0.31 & 0.24 \\
\hline
\end{tabular}


implicated in diabetic retinopathy and maculopathy [13] [15], circulating biomarkers in their use in diagnosis and prognosis remain weak. Our objective was to find out the relationship between serum levels of two biomarkers (VEGF-A and IL-6), the presence and severity of diabetic retinopathy.

Hyperglycemia causes metabolic changes that lead to oxidative stress, inflammation and vascular dysfunction, resulting in hyperpermeability, vascular occlusion and ischemia [17]. The median level of IL-6 and VEGF-A in our study was respectively of 33.5 (range 7.9 - 50.6) and 189.3 [range: 112.2 - 411.1]. The VEGF value is greater compared to the $34.5 \mathrm{pg} / \mathrm{ml}$ obtained in an Austrian study [18]. We found that the level of HbAlc was 8.5 [range: 7.8 - 10.3] in patients with diabetic retinopathy in our series. That can explain the high level of VEGF-A in patients with diabetic retinopathy in our series compared to the findings of the Austrian study where the median HBAlc was 7.5 (range: 5.3 10.6) [18].

The present study reveals that serum levels of VEGF-A are correlated to the presence of DR. Meanwhile there was no association between IL- 6 with the presence of DR. Il-6 is a cytokine produced by monocytes, macrophages, lymphocytes, fibroblasts and endothelial cells. VEGF is a potent vasoactive cytokine that increases vascular permeability and is involved in the development of diabetic retinopathy and diabetic maculopathy [19]. It affects the endothelial proteins of the tight junction, resulting in fluid extravasation and retinal oedema [20]. Many studies supported the hypothesis of association between vitreous inflammatory and angiogenic cytokines and their connection with the progression of diabetic retinopathy [11] [12]. But it is not clear whether the development of diabetic retinopathy can be linked to the circulating levels of these cytokines and whether they can be used as diagnostic tools. Moreover studies on this issue have reported contradictory results and only few or none of these investigations are conducted in Sub-Saharan Africa [14] [21] [22] [23].

In the present study, we found higher serum levels of VEGF-A in the DR group compared to the control group. This finding suggests that during the onset of diabetic retinopathy, a cascade of metabolic disorders like angiogenesis are activated and may lead to the progression of DR, in line with conclusions from a meta-analysis [24] that correlate VEGF to the presence of diabetic retinopathy. The expression of VEGF is stimulated by chronic hyperglycaemia increasing thus the vascular permeability in the retina.

We also found that there was a non-significant increase of IL-6 in the DR compared to the control group. This was also the case in a Belgium study [23]. Although inflammation plays a relevant role in the development of DR, serum concentration of IL-6 wasn't associated to it.

In a prospective Bulgarian study conducted on 38 healthy volunteers (group 1) and 39 patients with type $2 \mathrm{DM}$ (group 2), high but not significant levels were observed for diabetic patients compared to controls for VEGF $(p=0.067)$ and IL-6 ( $\mathrm{p}=0.132)$. Moreover, according to the severity of diabetic retinopathy, the 
level of VEGF ( $\mathrm{p}<0.0001)$ and Il-6 ( $\mathrm{p}<0.007)$ was significant higher in patients with proliferative diabetic retinopathy compared to those with non-proliferative diabetic retinopathy [14].

There was no relation between the 2 cytokines and the severity of DR. Moreover, no link was found between those circulating cytokines and the presence or lack of maculopathy. However, the relation between circulating VEGF and the severity of the diabetic retinopathy is found in the literature [13] [14], even though some contradictory findings are also reported [18]. Circulating IL-6 is also linked to the severity of the retinopathy according to the literature [14]. Our results can be explained by the laser coagulation used for patients with proliferative diabetic retinopathy. Laser coagulation can reduce the serum expression of these cytokines. In a study carried out in Egypt, there was a significant decrease in serum concentration of VEGF when the analysis was performed before and after laser coagulation in patients with proliferative diabetic retinopathy [25].

Patients with diabetes have stronger inflammation compared to healthy population. In our study, serum levels of IL-6 were higher in patients with uncontrolled diabetes ( $\mathrm{HbAlC} \geq 7 \%$ ). On the contrary, serum levels of VEGF were not associated to the level of glycosylated haemoglobin. These findings suggest that patients with high glycosylated haemoglobin values develop stronger inflammatory responses compared to the patients with controlled diabetes. This could be implicated in the pathogenesis of DR. Other cytokines as TNF-alpha was found to be linked to the level of glycosylated haemoglobin [14]. However, concerning the link between VEGF and uncontrolled diabetes, the results of the present study are contradictory to those found in the literature [14] [18].

Levels of cytokines (Il-6: $\mathrm{p}=0.31$; VEGF-A: $\mathrm{p}=0.24$ ) were not associated with the duration of diabetes in the present survey. These findings are in line with that of other authors [14]. This can be explained by the fact that the duration of diabetes as a well-known risk factor of the disease, was not associated to the presence of diabetic retinopathy in the present study $(\mathrm{p}=0.06$; multiple logistic regression).

The limit of our work lies in the size of the sample, particularly, the small number of patients that develop proliferative diabetic retinopathy. Further, literature is contradictory regarding the association between inflammatory and angiogenic cytokines and diabetic retinopathy. Sub-Saharan Africa does not present evidence about the connection between cytokines and diabetic retinopathy. Moreover, the use of fluorescein angiography could have brought more details for the classification of the diabetic retinopathy and the maculopathy. Prospective studies on a large number of patients are needed to understand whether these cytokines can be used either as a diagnostic tool or as a therapeutic tool or as both.

\section{Conclusion}

According to our findings, serum concentration of VEGF-A reflects the presence 
of diabetic retinopathy. Though IL-6 is associated to the imbalance of the diabetes, VEGF-A and IL- 6 are not correlated to the severity of diabetic retinopathy nor to the presence of maculopathy. These 2 biomarkers may play a role in the pathophysiology of diabetes and the diabetic retinopathy. Findings on Il-6 as on VEGF-A may therefore contribute to the development of the diagnosis tools and treatment of diabetes and diabetic retinopathy.

\section{Acknowledgements}

We thank the personnel from the laboratory of the National Obesity Center for their contribution to analysis.

\section{Contribution}

All authors made a substantial contribution to the concept or design of the work; or acquisition, analysis or interpretation of data, drafted the article or revised it critically for important intellectual content, and approved the version to be published.

\section{Conflicts of Interest}

The authors declare no conflicts of interest regarding the publication of this paper.

\section{References}

[1] Cho, N.H., Shaw, J.E., Karuranga, S., Huang, Y., da Rocha Fernandes, J.D., Ohlrogge, A.W. and Malanda, B. (2018) IDF Diabetes Atlas: Global Estimates of Diabetes Prevalence for 2017 and Projections for 2045. Diabetes Research and Clinical Practice, 138, 271-281. https://doi.org/10.1016/j.diabres.2018.02.023

[2] Yau, J.W.Y., Rogers, S.L., Kawasaki, R., Lamoureux, E.L., Kowalski, J.W., Bek, T., Chen, S.-J., Dekker, J.M., Fletcher, A., Grauslund, J., Haffner, S., Hamman, R.F., Ikram, M.K., Kayama, T., Klein, B.E.K., Klein, R., Krishnaiah, S., Mayurasakorn, K., O’Hare, J.P., Orchard, T.J., Porta, M., Rema, M., Roy, M.S., Sharma, T., Shaw, J., Taylor, H., Tielsch, J.M., Varma, R., Wang, J.J., Wang, N., West, S., Xu, L., Yasuda, M., Zhang, X., Mitchell, P. and Wong, T.Y. (2012) Global Prevalence and Major Risk Factors of Diabetic Retinopathy. Diabetes Care, 35, 556-564. https://doi.org/10.2337/dc11-1909

[3] Bourne, R.R.A., Stevens, G.A., White, R.A., Smith, J.L., Flaxman, S.R., Price, H., Jonas, J.B., Keeffe, J., Leasher, J., Naidoo, K., Pesudovs, K., Resnikoff, S. and Taylor, H.R. (2013) Causes of Vision Loss Worldwide, 1990-2010: A Systematic Analysis. The Lancet Global Health, 1, e339-e349. https://doi.org/10.1016/S2214-109X(13)70113-X

[4] Klein, B.E.K. (2007) Overview of Epidemiologic Studies of Diabetic Retinopathy. Ophthalmic Epidemiology, 14, 179-183.

https://doi.org/10.1080/09286580701396720

[5] Jingi, A.M., Noubiap, J.J.N., Ellong, A., Bigna, J.J.R. and Mvogo, C.E. (2014) Epidemiology and Treatment Outcomes of Diabetic Retinopathy in a Diabetic Population from Cameroon. BMC Ophthalmology, 14, 19. https://doi.org/10.1186/1471-2415-14-19 
[6] Lawan, A. and Mohammed, T. (2012) Pattern of Diabetic Retinopathy in Kano, Nigeria. Annals of African Medicine, 11, 75. https://doi.org/10.4103/1596-3519.93528

[7] Rotchford, A.P. and Rotchford, K.M. (2002) Diabetes in Rural South Africa-An Assessment of Care and Complications. South African Medical Journal, 92, 536-541.

[8] Simó, R. and Hernández, C. (2015) Novel Approaches for Treating Diabetic Retinopathy Based on Recent Pathogenic Evidence. Progress in Retinal and Eye Research, 48, 160-180. https://doi.org/10.1016/j.preteyeres.2015.04.003

[9] Stitt, A.W., Curtis, T.M., Chen, M., Medina, R.J., McKay, G.J., Jenkins, A., Gardiner, T.A., Lyons, T.J., Hammes, H.-P., Simó, R. and Lois, N. (2016) The Progress in Understanding and Treatment of Diabetic Retinopathy. Progress in Retinal and Eye Research, 51, 156-186. https://doi.org/10.1016/j.preteyeres.2015.08.001

[10] Aiello, L.P. (2005) Angiogenic Pathways in Diabetic Retinopathy. The New England Journal of Medicine, 353, 839-841. https://doi.org/10.1056/NEJMe058142

[11] Murugeswari, P., Shukla, D., Rajendran, A., Kim, R., Namperumalsamy, P. and Muthukkaruppan, V. (2008) Proinflammatory Cytokines and Angiogenic and Anti-Angiogenic Factors in Vitreous of Patients with Proliferative Diabetic Retinopathy and Eales' Disease. Retina, 28, 817. https://doi.org/10.1097/IAE.0b013e31816576d5

[12] Zhou, J., Wang, S. and Xia, X. (2012) Role of Intravitreal Inflammatory Cytokines and Angiogenic Factors in Proliferative Diabetic Retinopathy. Current Eye Research, 37, 416-420. https://doi.org/10.3109/02713683.2012.661114

[13] Gupta, N., Mansoor, S., Sharma, A., Sapkal, A., Sheth, J., Falatoonzadeh, P. and Kenney, M.C. (2013) Diabetic Retinopathy and VEGF. The Open Ophthalmology Journal, 7, 4. https://doi.org/10.2174/1874364101307010004

[14] Koleva-Georgieva, D.N., Sivkova, N.P. and Terzieva, D. (2011) Serum Inflammatory Cytokines IL- $1 \beta$, IL-6, TNF- $\alpha$ and VEGF Have Influence on the Development of Diabetic Retinopathy. Folia Medica (Plovdiv), 53, 44-50. https://doi.org/10.2478/v10153-010-0036-8

[15] Doganay, S., Evereklioglu, C., Er, H., Türköz, Y., Sevinc, A., Mehmet, N. and Şavli, H. (2002) Comparison of Serum NO, TNF- $\alpha$, IL- $1 \beta$, sIL-2R, IL-6 and IL-8 Levels with Grades of Retinopathy in Patients with Diabetes Mellitus. Eye, 16, 163. https://doi.org/10.1038/sj/eye/6700095

[16] Wilkinson, C.P., Ferris, F.L., Klein, R.E., Lee, P.P., Agardh, C.D., Davis, M., Dills, D., Kampik, A., Pararajasegaram, R., Verdaguer, J.T., Global Diabetic Retinopathy Project Group (2003) Proposed International Clinical Diabetic Retinopathy and Diabetic Macular Edema Disease Severity Scales. Ophthalmology, 110, 1677-1682. https://doi.org/10.1016/S0161-6420(03)00475-5

[17] Brownlee, M. (2005) The Pathobiology of Diabetic Complications: A Unifying Mechanism. Diabetes, 54, 1615-1625. https://doi.org/10.2337/diabetes.54.6.1615

[18] Zehetner, C., Kirchmair, R., Kralinger, M. and Kieselbach, G. (2013) Correlation of Vascular Endothelial Growth Factor Plasma Levels and Glycemic Control in Patients with Diabetic Retinopathy. Acta Ophthalmologica, 91, e470-e473. https://doi.org/10.1111/aos.12081

[19] Simo, R. and Hernandez, C. (2008) Intravitreous Anti-VEGF for Diabetic Retinopathy: Hopes and Fears for a New Therapeutic Strategy. Diabetologia, 51, 1574. https://doi.org/10.1007/s00125-008-0989-9

[20] Antonetti, D.A., Barber, A.J., Hollinger, L.A., Wolpert, E.B. and Gardner, T.W. (1999) Vascular Endothelial Growth Factor Induces Rapid Phosphorylation of Tight 
Junction Proteins Occludin and Zonula Occluden 1. A Potential Mechanism for Vascular Permeability in Diabetic Retinopathy and Tumors. The Journal of Biological Chemistry, 274, 23463-23467. https://doi.org/10.1074/jbc.274.33.23463

[21] Le, D.S.N.T., Miles, R., Savage, P.J., Cornell, E., Tracy, R.P., Knowler, W.C. and Krakoff, J. (2008) The Association of Plasma Fibrinogen Concentration with Diabetic Microvascular Complications in Young Adults with Early-Onset of Type 2 Diabetes. Diabetes Research and Clinical Practice, 82, 317-323.

https://doi.org/10.1016/j.diabres.2008.08.019

[22] Lee, I.G., Chae, S.L. and Kim, J.C. (2006) Involvement of Circulating Endothelial Progenitor Cells and Vasculogenic Factors in the Pathogenesis of Diabetic Retinopathy. Eye (London, England), 20, 546-552.

https://doi.org/10.1038/sj.eye.6701920

[23] Ozturk, B.T., Bozkurt, B., Kerimoglu, H., Okka, M., Kamis, U. and Gunduz, K. (2009) Effect of Serum Cytokines and VEGF Levels on Diabetic Retinopathy and Macular Thickness. Molecular Vision, 15, 1906-1914.

[24] Zhou, Z., Ju, H., Sun, M. and Chen, H. (2019) Serum Vascular Endothelial Growth Factor Levels Correlate with Severity of Retinopathy in Diabetic Patients: A Systematic Review and Meta-Analysis. Disease Markers, 2019, Article ID: 9401628. https://doi.org/10.1155/2019/9401628

[25] Mahdy, R.A. and Nada, W.M. (2011) Evaluation of the Role of Vascular Endothelial Growth Factor in Diabetic Retinopathy. Ophthalmic Research, 45, 87-91. https://doi.org/10.1159/000317062 